\title{
Cataract Surgery with Systemic Diseases: Is Perioperative Management Necessary?
}

\author{
Devika S Joshi ${ }^{*}$, Shrikant D Joshi MS ${ }^{2}$ \\ ${ }^{1}$ Panel Consultant, Deenanath Mangeshkar Hospital and Research Centre, Pune, India \\ ${ }^{2}$ Honorary Consultant, Deenanath Mangeshkar Hospital and Research Centre, Pune, India \\ *Corresponding Author: Devika S Joshi, Panel Consultant, Deenanath Mangeshkar Hospital and Research \\ Centre, Pune, India; Email: devika.joshi1788@gmail.com \\ Received Date: 04-05-2021; Accepted Date: 25-05-2021; Published Date: 02-06-2021 \\ Copyright $^{\circledR} 2021$ by Joshi DS, et al. All rights reserved. This is an open access article distributed under the terms \\ of the Creative Commons Attribution License, which permits unrestricted use, distribution, and reproduction in \\ any medium, provided the original author and source are credited.
}

\begin{abstract}
Background: Cataract Surgery is commonly done surgery in the geriatric age group. These patients may have several systemic diseases to which adequate attention must be paid during surgical planning, local anesthesia administration and in the postoperative period. This study aimed to find the actual need for any anesthetic intervention during surgery, pertaining to the patient's systemic condition.
\end{abstract}

Objectives: To assess perioperative management required for patients who have undergone cataract surgeries with respect to (w.r.t.) specific systemic comorbidities.

Methods: Hospital records of 2022 patients who had undergone cataract surgery over two years were analyzed retrospectively. 519 patients were found to have one or more systemic comorbidities and were included in this study. Data were analyzed to determine perioperative management (monitoring and interventions) required for these patients w.r.t. their systemic comorbidities.

Results: 320 patients $(61.7 \%)$ were males and 199 (38.3\%) were females. Various systemic diseases were observed: cardiovascular 166 (28.6\%) (most common), hypertension 143 (24.7\%), neurological $56(9.1 \%)$, malignancies $39(6.7 \%)$, renal $24(4.1 \%)$, and respiratory 37 (6.4\%). $511(98.5 \%)$ patients did not require any preoperative interventions. 30 patients $(5.8 \%)$ required intraoperative interventions like some intravenous drug injections. 2 patients $(0.2 \%)$ required the postoperative intervention of Tablet Sorbitrate for chest pain and physician's 
consultation. $17(3.3 \%)$ required sedation during surgery. None required overnight hospitalization.

Conclusion: Most patients with systemic comorbidities who undergo cataract surgery under local anesthesia do not require perioperative management, with an anesthesiologist standby. Such surgeries may be safely carried out at standalone centers in a resource-limited setup with an anesthesiologist standby.

\section{Keywords}

Cataract Surgery; Anesthesia; Systemic Disease; Comorbidity

\section{Introduction}

Increased longevity and life expectancy have led to an increase in the number of cataract surgeries being performed today. Lifestyle changes along with many other factors are contributing to a rise in systemic co-morbidities like hypertension, diabetes, stroke, Ischemic Heart Disease (IHD) and Coronary Artery Bypass Grafts (CABG), neurological, renal disorders, blood dyscrasias, as well as malignancies [1,2]. The anesthesia required for cataract surgery is either local or topical with minimal systemic effects. However, it has been observed that ophthalmologists prefer if these "high risk" groups of patients undergo cataract surgeries at a tertiary care hospital with facilities for stand by anesthesiologists and additional support like ICU, blood bank and dialysis rather than at standalone clinics with none of the abovementioned facilities. Our study aims to determine whether any special perioperative management is required for patients with systemic comorbidities. We hope that this study would contribute to a large extent in allaying the fears in the minds of ophthalmic surgeons about operating the so-called "high risk" patients at small centers, while at the same time reducing the burden of referred "routine" cataract cases on tertiary centers. Similarly, this study hopes to ascertain locally relevant suggestions as to which patients with systemic comorbidities need to be definitely referred to a tertiary care facility for cataract surgery.

\section{Methodology}

A retrospective data analysis was conducted in the department of ophthalmology at a multispecialty hospital in Western Maharashtra. Hospital records from the past two years of patients undergoing cataract surgery were studied to determine the perioperative management (monitoring and interventions) required for these patients with respect to their specific systemic co-morbidities. Records of 2022 patients who had undergone cataract surgery in the past two years were retrieved and checked each for the presence of coexistent systemic disease or comorbidity. Data of 519 patients with systemic disease, thus retrieved, was entered into and 
analyzed using SPSS version 23. Quantitative data has been expressed as arithmetic mean and standard deviations, while categorical data have been expressed as frequencies and percentages.

\section{Results}

It was seen from the records that all of the 519 patients with systemic co-morbidities had undergone routine laboratory investigations including hemogram, fasting and post-prandial blood sugar levels, routine and microscopic examination of urine and ECG. The treating physicians had certified the preoperative fitness with respect to their co-morbidities. Patients were asked to continue or discontinue or change their routine medications as advised by their Physician. No additional investigations or assessments were requested by the operating surgeon. Cataract surgeries were carried out by different expert surgeons with at least 10-15 years of experience in ophthalmic practice and performing at least 300 cataract surgeries per year. All the cataract surgeries were carried out under local anesthesia (peribulbar/sub-tenons) administered by the operating surgeon with a qualified and experienced anesthesiologist present on standby. Local anesthesia comprised of a total 8-10 ml of 1:1 mixture in which $2 \%$ Lignocaine with 1:2,00,000 Adrenaline and Bupivacaine 0.5\% (mixed with Hyaluronidase (50 $\mathrm{IU} / \mathrm{ml}$ ) was administered. Any intervention required in the pre-operative, intraoperative or postoperative period, was carried out by the anesthesiologist present at that time based on the patient's assessment. Intraoperatively all patients had a secure intravenous line in place. All patients were monitored with a pulse oximeter and Sphygmomanometry. On a case-to-case basis, intraoperative ECG (for a patient with known cardiac ailment), Blood sugar (known Diabetic patient) etc. were also monitored by the anesthesiologist.

There were 320(61.7\%) were males and 199(38.3\%) females. Maximum patients (40.5\%) were in the age group of 60-70 years followed by $70-80$ years $(37.8 \%)$. The mean age of participants was 68 years.

The different co-morbidities encountered in our study were divided into categories as follows: cardiology (post-CABG, angioplasty, atherosclerosis, coronary artery diseases, cardiac arrhythmias, myocardial infarction, chronic ischemic heart disease, cardiomyopathies, valvular heart diseases, congestive cardiac failure), hematology and oncology (patients with ongoing and completed treatments for different neoplasia, multiple metastases, severe anemia, idiopathic thrombocytopenic purpura, low platelet counts, and multiple myeloma), thyroid disorders (hyperthyroidism and Graves' disease), renal disorders (chronic renal failure, renal transplantation, diabetic nephropathy, patients on dialysis, neurology (old and new-onset stroke, epilepsy, parkinsonism, Alzheimer's disease), respiratory conditions (asthma, COPD).

Fig. 1 (color pie chart) shows the distribution of systemic co-morbidities among the 519 patients. It was seen that $166(32 \%)$ patients had a cardiac co-morbidity followed by hypertension in $143(27.6 \%)$, neurological conditions in $56(10.8 \%)$, cancer in $39(7.5 \%)$, renal in $24(4.6 \%)$ patients. 
Other patients included in combined disorders, had hematological disorders (2.2\%), Diabetes (1.7\%), Spine disorders (1.7\%), psychiatric disorders (schizophrenia) (0.5\%) and other disorders like genetic (Down's syndrome), connective tissue disorders (rheumatoid arthritis, systemic lupus erythematosus), skin disorders.

Out of all the disorders, the majority $53 \%$ of individuals belonged to the cardiac, hypertension and vascular disorder group while the least common was skin and psychiatric disorders $(0.5 \%$ each).

Despite India heading to be the diabetes capital of the world, the number of diabetes-only patients in this study is very less $(1.7 \%)$ as only those diabetics with good glycemic control were posted for cataract surgery. Newly diagnosed and poorly controlled diabetics were excluded from the study. Diabetic complications were included in the respective general systemic distribution e.g. diabetic nephropathy was included in renal disorders.

In the preoperative period, 8 patients underwent additional preoperative monitoring including immediate preoperative repeat BSL measurement as the patient was fragile diabetic and repeat ECG as new ectopics were noted. In some individuals, preoperative interventions required were having a bronchodilator inhaler on standby, an adjustment in the dose of insulin for glycemic control, preoperative tablet midazolam for supraventricular tachycardia, Injection (Inj) amoxicillin/clavulanic acid as per surgeon's request and Injection ondansetron as the patient had an episode of vomiting (Table 1-4).

In our study, all patients underwent cataract surgery under local anesthesia administered by the operating surgeon with an anesthesiologist on standby. The majority of patients (>90\%) did not require any preoperative, intraoperative or postop interventions by the Anesthesiologist. 17 (3.3\%) individuals required administration of Inj Midazolam as a sedative. These intraoperative interventions were not in view of the patient's co-morbidity. They were carried out based on the anesthesiologist's clinical judgment at that time point (Table 5).

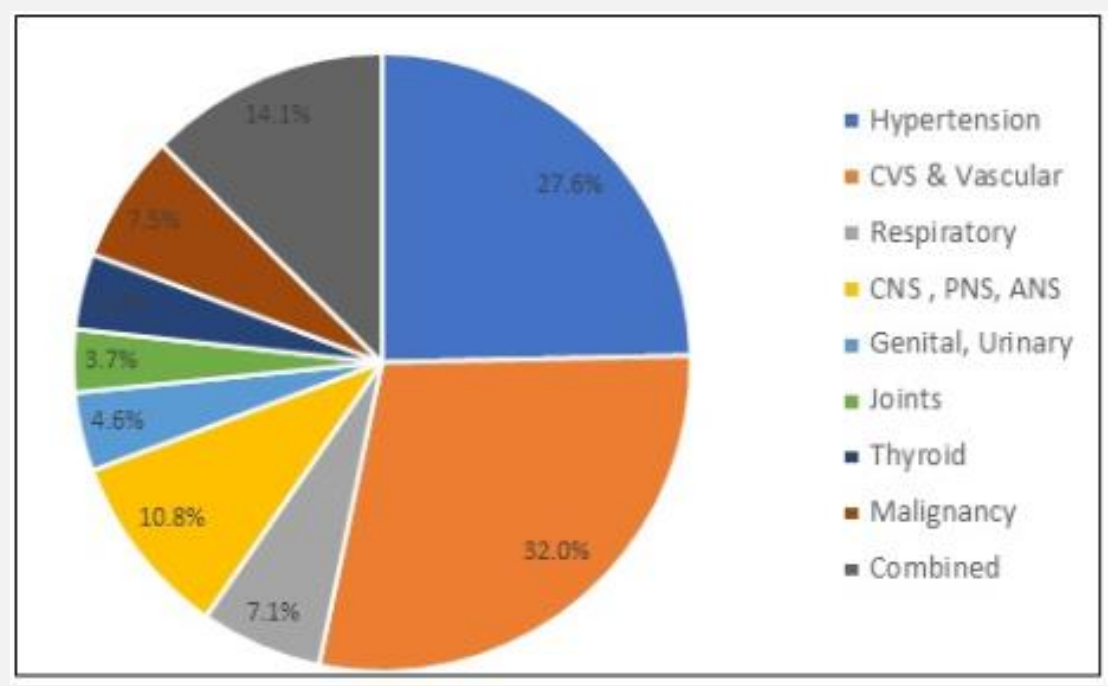

Figure 1: Pie chart with percentage frequency of systemic diseases. 


\begin{tabular}{|c|c|c|c|c|}
\hline Period & $\begin{array}{c}\text { Investigation/Intervention } \\
\text { (YES) }\end{array}$ & $\%$ & $\begin{array}{c}\text { Investigation/Intervention } \\
\text { (NO) }\end{array}$ & $\%$ \\
\hline Preoperative & 8 & 1.5 & 511 & 98.5 \\
\hline Intraoperative & 30 & 5.8 & 489 & 94.2 \\
\hline Postoperative & 2 & 0.4 & 517 & 99.6 \\
\hline
\end{tabular}

Table 1: Pre/Intra/Postoperative interventions.

\begin{tabular}{|c|c|c|}
\hline Treatment & Frequency & Percentage \\
\hline $\begin{array}{c}\text { BSL intra op 221mg/dl } \\
\text { Surgery not deferred }\end{array}$ & 1 & 0.2 \\
\hline ECG & 1 & 0.2 \\
\hline Bronchodilator inhaler kept stand by & 1 & 0.2 \\
\hline Inj amoxicillin/clavulanic acid 1.25mg & 1 & 0.2 \\
\hline Inj Ondansetron & 1 & 0.2 \\
\hline nil & 511 & 98.5 \\
\hline pre op insulin dose reduced & 1 & 0.2 \\
\hline Tab Midazolam was given pre op i/v/o SVT & 1 & 0.2 \\
\hline Total & 1 & 0.2 \\
\hline
\end{tabular}

Table 2: Preoperative interventions.

\begin{tabular}{|c|c|c|}
\hline Treatment & Frequency & Percent \\
\hline Inj Atropine & 1 & 0.2 \\
\hline Inj Metoprolol 0.5mg+0.5mg & 1 & 0.2 \\
\hline Inj Metoprolol + inj midazolam & 2 & 0.4 \\
\hline Inj Glycopyrrolate 0.2mg & 1 & 0.2 \\
\hline inj Midazolam & 24 & 4.6 \\
\hline Inj Midazolam, inj Ranitidine, inj Ondansetron & 1 & 0.2 \\
\hline nil & 489 & 94.2 \\
\hline Total & 519 & 100 \\
\hline
\end{tabular}

Table 3: Intraoperative interventions.

\begin{tabular}{|c|c|c|}
\hline Postoperative Intervention & Frequency & Percent \\
\hline c/o chest pain received sorbitrate & 1 & 0.2 \\
\hline Physician reference & 1 & 0.2 \\
\hline NIL & 517 & 99.6 \\
\hline
\end{tabular}

Table 4: Postoperative interventions. 


\begin{tabular}{|c|c|c|}
\hline \multicolumn{2}{|c|}{ Frequency } & Percent \\
\hline LAonly, No sedation & 502 & 96.7 \\
\hline LA + sedation & 17 & 3.3 \\
\hline Total & 519 & 100 \\
\hline
\end{tabular}

Table 5: Anesthesia requirement for comorbidity.

\section{Discussion}

Increased longevity and life expectancy have led to an increase in the number of cataract surgeries being performed nowadays. This is coupled with a rise in systemic co-morbidities like hypertension, diabetes, stroke, CABGs, neurological and renal disorders, blood dyscrasias, as well as malignancies [2].

Patients with cataracts generally tend to be elderly and may have one or more coexisting systemic morbidities. Many ophthalmologists feel that a thorough medical examination with laboratory investigations should be conducted before a patient can be considered for cataract surgery [3]. On the other hand, others feel that such tests are not required but order them anyway because of one or many of the following reasons: institutional requirements, medicolegal concerns or a belief that another physician may have wanted them performed $[4,5]$. The study by Schein OD, et al., reports no difference observed between the rates of perioperative medical events, including such events as hospitalization and death, which were found to be the same in patients who underwent a standard battery of laboratory tests as against those who did not undergo the same tests before cataract surgery. Also, there was no significant difference when stratified according to specific comorbid conditions or even the severity of the condition. Perioperative death or hospitalization was in fact very rare with most of the medical events taking place during the surgery and not being serious [6,7]. Also, the American Academy of Family Physicians (AAFP) 2013 recommends that Patients in their usual state of health who are undergoing cataract surgery do not require preoperative testing [8].

The 2016 American Academy of Ophthalmology Preferred Practice Pattern (PPP) recommends patient monitoring during the administration of anesthesia and surgery including using a heart monitor, pulse oximetry, and measurement of blood pressure and respiration performed by trained personnel such as an anesthesiologist or registered nurses or respiratory therapists [9].

Schein OD, et al., in 2000 showed that there was no significant difference in the safety of cataract surgery in patients with thorough preoperative evaluation and those without preoperative investigations [4]. Eghrari, et al., reported 2 case reports in cataract surgery in patients with left ventricular assisted devices. They showed that there were no systemic complications and the surgery was uneventful. The surgery was carried out in a supine position with an anesthesiologist and a perfusionist on standby and no intervention was needed in these patients [10]. Our study has similar findings, in which out of 166 patients with cardiac disorders only $5(3.01 \%)$ required anesthetic intervention. Zawar S and Kolte R similarly reported that

Joshi DS | Volume 2; Issue 2 (2021) | JOAR-2(2)-015 | Research Article

Citation: Joshi DS, et al. Cataract Surgery with Systemic Diseases: Is Perioperative Management

Necessary? J Ophthalmol Adv Res. 2021;2(2):1-10.

DOI: http://dx.doi.org/10.46889/JOAR.2021.2203 
cataract surgery in patients with long-standing cardiac diseases was uneventful with no interventions necessary. These patients were those who were red-flagged as high-risk patients by their physicians. These surgeries were performed under local anesthesia with an anesthesiologist on standby. In fact, general anesthesia was not indicated in these patients due to hemodynamic instability [11].

The 2007 guidelines on perioperative risk assessment from the American College of Cardiology and American Heart Association (ACC/AHA) do not recommend ECG in asymptomatic patients undergoing low-risk non-cardiac procedures regardless of the patient's age [12]. A randomized study carried out at Johns Hopkins University assessed the value of routine preoperative medical testing in patients scheduled for elective cataract surgery. It was noted that there was no difference in the overall rate of complications between the two groups that is, those preceded or not preceded by a standard set of tests including Electrocardiogram (ECG), Complete Blood Count (CBC), serum electrolytes, blood urea nitrogen, serum creatinine and blood glucose [4].

Some of the common conditions for which patients with cataracts get labeled "high risk" are significant arrhythmia like symptomatic bradycardia, unstable coronary syndromes including recent myocardial infarcts, decompensated heart failure, severe valvular disease and necessitate evaluation before surgery [13]. The risk in cataract surgery is about vasovagal or anaphylactic shock after giving peribulbar or retrobulbar block. Also, a patient's anxiety about the surgery and oculocardiac reflex may affect a heart that is already compromised and lead to further systemic complications or even death. Many rural or tribal patients requiring cataract surgeries are unable to afford certain cardiac medications and special preoperative investigations. They may eventually be lost to follow up and gradually become blind [10]. In this study, intraoperatively, one patient required Midazolam for Ventricular tachycardia and only two patients required post-operative intervention which included administration of Tab Sorbitrate for chest pain and physician's consultation for newly detected ectopic beats in the postoperative period. This was not related to the existing systemic co-morbidity and were new events.

Thus, as per the recently revised European Society of Cardiology (ESC) and the European Society of Anesthesiology (ESA) Guidelines 2015 for non-cardiac surgery, patients with stable CVD undergoing low risk (which includes Eye surgery) do not require additional preoperative cardiac assessment. On the other hand, patients with a known or high risk of cardiac disease scheduled for high-risk non-cardiac surgery should undergo a cardiac assessment by a multidisciplinary expert team. Preoperative resting ECG is not recommended in asymptomatic patients without risk factors undergoing low-risk surgery [14].

As noted by Eghrari AO, et al., cataract surgery can be safely performed in terminally ill patients with advanced cardiac failure or awaiting cardiac transplantation on Left Ventricular Assist Devices (LVADs) in the presence of adequate monitoring resources [9]. Recommendations include extensive preoperative cardiology evaluations and continuation of warfarin through the day of surgery. These authors have documented the use of monitored sedation using fentanyl and midazolam. 
In Chronic Renal Failure (CRF) patients, there is a high risk of pre-and postoperative complications [15]. In a study by Chen Y, et al., the authors stopped heparin before surgery and started hemostatic drug in CRF patients [16]. As compared to older techniques, Phacoemulsification and intraocular lens implantation are effective and safe in non-diabetic CRF patients with cataract on maintenance dialysis if these patients have controlled blood pressure within normal range. In this study, one patient with CRF required Injection Metoprolol intraoperatively to control rising blood pressure.

In a study in Palermo, Italy, the authors noted the predictive role of psychiatric disorders as determinants of hospitalization inappropriateness in cataract surgery. They also found a negative correlation between inappropriate hospitalization and conditions such as hypertension and diabetes. They concurred that this was because these common diseases in the elderly population are effectively addressed [17]. Our study has similar findings as none of the patients included required overnight hospital admissions.

In our study, 17 (3.3\%) individuals required administration of Inj Midazolam as a sedative. These intraoperative interventions were not in view of the patient's co-morbidity. They were carried out based on the anesthesiologist's clinical judgment at that time point. On a case-tocase basis, anxious or uncooperative patients may require sedation in addition to regional anesthesia. Discussing the peri-operative process with the patient prior to surgery may help alleviate anxiety and avoid the need for sedation [18].

Literature regarding guidelines for operating a patient for cataract in the presence of specific coexisting systemic comorbidities is virtually non-existent with only a few publications reporting experiences with a variety of systemic comorbidities. Rosenfeld, et al., in their study noted that intervention by anesthesia personnel was required in more than one-third of 1006 consecutive cataract surgery cases. Several of the patients studied had hypertension, cardiovascular diseases, pulmonary disease etc. As the authors could not reliably predict those patients at risk, they stated that monitored anesthesia care seemed justified in cataract surgery with the patient under local anesthesia [19].

In a recent study by Koolwijk J, et al., the authors concluded that cataract surgery can be safely performed in an outpatient clinic, in the absence of the anesthesia service and with limited workup and monitoring. Basic first aid and basic life support skills seem to suffice in case of an adverse event [20].

Pepin M, et al., observed that it is necessary to have selection criteria for patients who undergo cataract surgery under topical anesthesia without the anesthesia team. This way, organizational constraints are reduced while still ensuring patient safety. However, in patients with unstable disease or risk of agitation, it is better that the Anesthesiologist is present to optimize the medications administered during surgery [21].

Similarly, the authors feel that it is important to draw reliable conclusions based on a large number of cases of cataract operated in patients with a variety of coexistent systemic

Joshi DS | Volume 2; Issue 2 (2021) | JOAR-2(2)-015 | Research Article

Citation: Joshi DS, et al. Cataract Surgery with Systemic Diseases: Is Perioperative Management

Necessary? J Ophthalmol Adv Res. 2021;2(2):1-10.

DOI: http://dx.doi.org/10.46889/JOAR.2021.2203 
morbidities and try and establish locally relevant recommendations as to which patients need the monitoring and intervention facilities provided by a tertiary care center.

\section{Conclusion}

Cataract surgeries can be done safely in a standalone or nuclear setup with monitored anesthesia care without other support viz ICU, blood bank and without a multidisciplinary backup. We suggest that standard routine preoperative tests including hemogram, random blood sugar, and ECG should be advised in all patients.

Patients with known preoperative comorbidities such as ischemic heart disease should be referred to the concerned specialist to assess preoperative risks. It may be wise in centers without ICU facilities and blood banks to perform cataract surgery under local anesthesia with an anesthesiologist on standby for added safety.

\section{Acknowledgements}

Thanks to Dr Asawari Kanade for help with the statistical analysis and Dr Gauri Oka for fine tuning the manuscript content. Also, a big thanks to Dr Shweta A. Kelkar, Pune for data collection.

\section{References}

1. Khera AV, Emdin CA, Drake I, Natarajan P, Bick AG, Cook NR, et al. Genetic risk, adherence to a healthy lifestyle, and coronary disease. N Engl J Med. 2016;375:2349-58.

2. Yanping L, Schoufour J, Wang DD, Dhana K, Pan A, Liu X, et al. Healthy lifestyle and life expectancy free of cancer, cardiovascular disease, and type 2 diabetes: prospective cohort study. BMJ. 2020;368:I6669.

3. Chen CL, Lin GA, Bardach NS, Clay TH, Boscardin WJ, Gelb AW, et al. Preoperative medical testing in Medicare patients undergoing cataract surgery. N Engl J Med. 2015;372(16):1530-8.

4. Schein OD, Katz J, Bass EB, Tielsch JM, Lubomski LH, Feldman MA, et al. The value of routine preoperative medical testing before cataract surgery. N Engl J Med. 2000;342:168-75.

5. Bass EB, Steinberg EP, Luthra R, Schein OD, Tielsch JM, Javitt JC, et al. Do ophthalmologists, anesthesiologists, and internists agree about preoperative testing in healthy patients undergoing cataract surgery? Arch Ophthalmol. 1995;113(10):1248-56.

6. Keay L, Lindsley K, Tielsch J, Katz J, Schein O. Routine preoperative medical testing for cataract surgery. Cochrane Database Syst Rev. 2012;3(3):CD007293.

7. Keay L, Lindsley K, Tielsch J, Katz J, Schein O. Routine preoperative medical testing for cataract surgery. Cochrane Database Syst Rev. 2019;1(1):CD007293.

8. Feely MA, Collins CS, Daniels PR, Kebede EB, Jatoi A, Mauck KF. Preoperative testing before noncardiac surgery: guidelines and recommendations. Am Fam Physician. 2013;87:414-8.

9. Olson RJ, Braga-Mele R, Chen SH, Miller KM, Pineda R, Tweeten JP, et al. Cataract in the adult eye preferred practice pattern®. Ophthalmology. 2017;124(2):1-19. 
10. Eghrari AO, Rivers RJ, Alkharashi M, Rajaii F, Nyhan D, Sikder S. Cataract surgery in patients with left ventricular assist device support. J Cataract Refractive Surg. 2014;40(4):675-8.

11. Zawar S, Kolte R. Pain-free cataract surgery in patients with cardiac risk. Ind J Ophthalmol. 2012;60(2):157.

12. Fleisher LA, Beckman JA, Brown KA, Calkins H, Chaikof EL, Fleischmann KE,et al. Guidelines on perioperative cardiovascular evaluation and care for noncardiac surgery: a report of the American college of cardiology/American heart association task force on practice guidelines. $\mathrm{J}$ Am Coll Cardiol. 2007;50(17):e159-242.

13. De Hert S, Imberger G, Carlisle J, Diemunsch P, Fritsch G, Moppett I, et al. Task force on preoperative evaluation of the adult noncardiac surgery patient of the European society of anaesthesiology. Preoperative evaluation of the adult patient undergoing non-cardiac surgery: guidelines from the European society of anaesthesiology. Euro J Anaesthesiol. (EJA). 2011;28(10):684-722.

14. Guarracino F, Baldassarri R, Priebe HJ. Revised ESC/ESA guidelines on non-cardiac surgery: cardiovascular assessment and management. Implications for preoperative clinical evaluation. Minerva Anestesiol. 2015;81(2):226-33.

15. Dursun D, Akova YA, Akman A, Oto S, Aydin P. Complications of extracapsular cataract surgery in chronic renal failure patients. Eye. 2000;14(2):176-9.

16. Chen Y, Cai J, Liu H. Phacoemulsification and intraocular lens implantation in patients with chronic renal failure and cataract. [Zhonghua yan ke za zhi] Chinese J Ophthalmol. 2001;37(5):332-4.

17. Cillino S, Iggui A, Di Naro S, Cillino G, Matranga D, Mazzucco W, et al. Determinants of inappropriate hospitalization in cataract surgery in the south of Italy: a retrospective study. Intl Ophthalmol. 2019;39(4):873-81.

18. Kumar CM, Seet E, Eke T, Irwin MG, Joshi GP. Peri-operative considerations for sedation-analgesia during cataract surgery: a narrative review. Anaesthesia. 2019;74(12):1601-10.

19. Rosenfeld SI, Litinsky SM, Snyder DA, Plosker H, Astrove AW, Schiffman J. Effectiveness of monitored anesthesia care in cataract surgery. Ophthalmol. 1999;106(7):1256-61.

20. Koolwijk J, Fick M, Selles C, Turgut G, Noordergraaf JI, Tukkers FS, et al. Outpatient cataract surgery: incident and procedural risk analysis do not support current clinical ophthalmology guidelines. Ophthalmol. 2015;122(2):281-7.

21. Pepin M, Rineau E, Caignard A, Leruez S, Gohier P. Topical anesthesia without the use of an anesthesiologist in adult outpatient cataract surgery: Assessment of selection criteria on consultation, report of 248 cases. J Francais d'Ophtalmologie. 2018;41(5):441-6. 